Case

\title{
Surgical Resection of a Solitary Pulmonary Metastasis from Basaloid Squamous Cell Carcinoma of the Esophagus: A Case Report
}

\author{
Takayuki Kosaka, MD, PhD, Akira Mogi, MD, PhD, Ei Yamaki, MD, PhD, \\ Tatsuya Miyazaki, MD, PhD, and Hiroyuki Kuwano, MD, PhD
}

\begin{abstract}
Basaloid squamous cell carcinoma (BSCC) of the esophagus is a relatively rare variant of esophageal malignancies. It is regarded as a disease with a poor prognosis because of the high frequency of distant metastases. We managed a case of resected solitary pulmonary metastasis of BSCC of the esophagus in a 78-year-old female. We performed curative thoracic esophagectomy with three-field lymph node dissection for her disease. After two years and nine months, a thoracoscopic partial resection of the left lung was performed for the solitary pulmonary metastasis. At present, one year after the surgery, the patient is doing well without any recurrence. There have been few reports of case of resected pulmonary metastases. In addition, no treatment strategy for pulmonary metastases from BSCC of the esophagus has been established because of the limited number of cases. Additional cases are required to determine the treatment method.
\end{abstract}

Keywords: basaloid squamous cell carcinoma, pulmonary metastasis, esophageal cancer

\section{Introduction}

Basaloid squamous cell carcinoma (BSCC) of the esophagus is a relatively rare variant of esophageal malignancies. It is regarded as a disease with a poor prognosis because of the high frequency of distant metastases. There have been few reports of cases of resected pulmonary metastases. In this study, we report a case of a resected solitary pulmonary metastasis of BSCC of the esophagus.

Department of General Surgical Science, Graduate School of Medicine, Gunma University, Maebashi, Gunma, Japan

Received: May 1, 2013; Accepted: June 26, 2013

Corresponding author: Takayuki Kosaka, MD, PhD. Department of General Surgical Science, Gunma University Graduate School of Medicine, 3-39-22 Showa-machi, Maebashi, Gunma 371-8511, Japan

Email: tkosaka@gunma-u.ac.jp

(C)2014 The Editorial Committee of Annals of Thoracic and Cardiovascular Surgery. All rights reserved.

\section{Case Report}

A 78-year-old female visited our hospital because of an uncomfortable feeling during meals. An upper gastrointestinal endoscopy showed a tumorous lesion in the esophagus, and histopathological examination of biopsy specimens suggested BSCC. Preoperative examination revealed that clinical stage was cT3N2M0, stage III. Computed tomography (CT) scans revealed a mammary gland tumor at the same time. First, curative thoracic esophagectomy with three-field lymph node dissection was performed. It was pathologically determined as BSCC (Fig. 1), and the pathological stage was pT3N2M0, stage III. A month later, the mammary gland tumor was diagnosed as breast cancer, and mastectomy was performed to treat the synchronous breast cancer. Pathologically, it was an invasive breast ductal cancer, and the pathological stage was pT2N0M0, stage IIA. Because the pathological stage of esophageal cancer was III, docetaxel was administered as adjuvant therapy. After a 


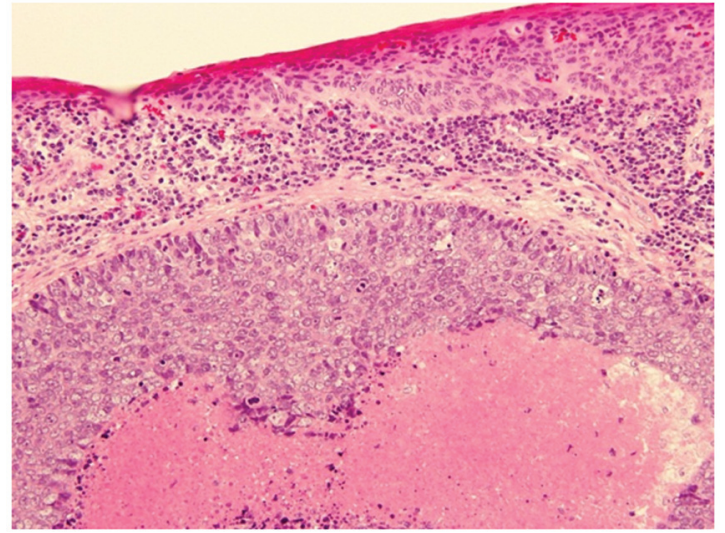

Fig. 1 Histological observation of esophageal tumor showed that the tumor cells, which were similar to basaloid cells with enlarged nuclei, formed tumor cell nests of different sizes, which grew and infiltrated in a solid, cord-like arrangement. In addition, large necrotic foci were found in the center of tumor cell nests, and the deposition of acidophilic basement membrane-like substances was observed between tumor cells. Pathological examination determined the cells as basaloid squamous cell carcinomaBSCC.

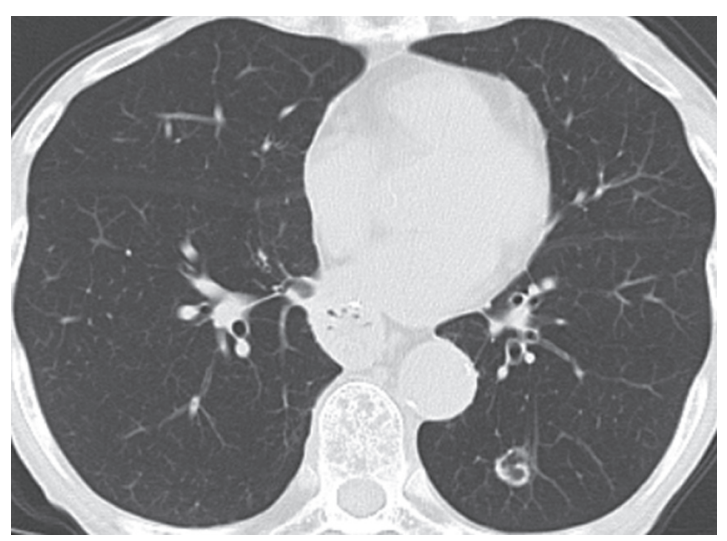

Fig. 2 Chest computed tomography (CT) at two months after the initiation of treatment with TS-1.

year and four months, enlargement of mediastinal lymph nodes was detected; this was diagnosed as recurrence of esophageal cancer. The area was irradiated with a dose of $60 \mathrm{~Gy}$, and a complete response was obtained.

After 2 years and 4 months, a CT scan detected a 5-mm nodule in the lower lobe of the left lung. Because it was difficult to obtain a qualitative diagnosis, we decided on follow-up observation of the lesion. After
(A)

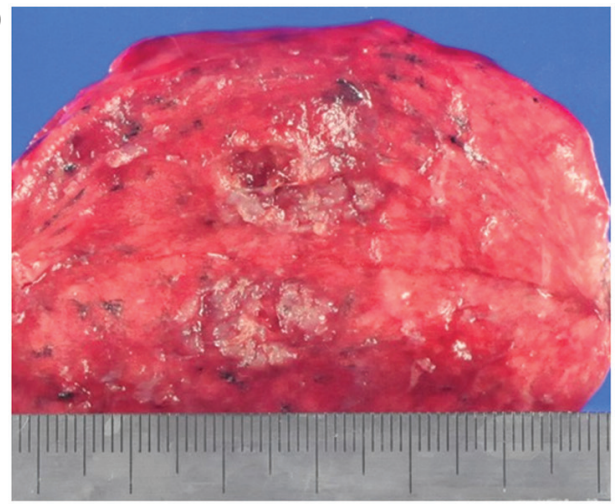

(B)

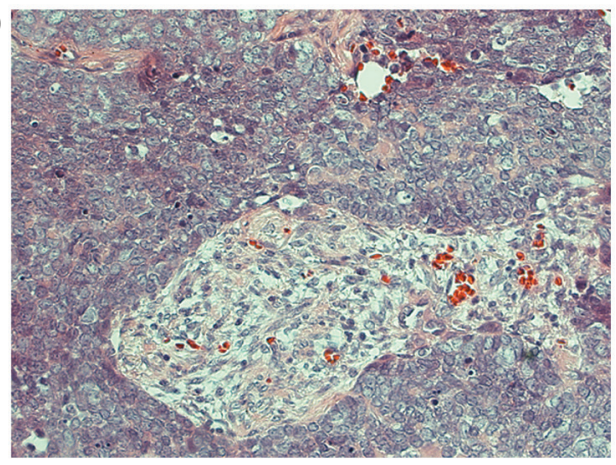

Fig. 3 (A) Macroscopic observation showed a white nodule with indistinct borders. (B) Histological observation of pulmonary nodule showed that atypical cells, similar to stratified squamous epithelium basal cells with a high nuclear cytoplasmic (N/C) ratio, formed tumor cell nests of different sizes that grew to replace the lung parenchyma and were accompanied by stroma fibrosis. Pathological examination led to the diagnosis of pulmonary metastasis from basaloid squamous cell carcinomaBSCC of the esophagus.

three months, a follow-up CT found that the nodule had enlarged to $9 \mathrm{~mm}$, and it was determined as a metastasis. The patient was then administered oral TS- 1 because the primary tumor could have been either esophageal or breast cancer. Two months after the initiation of treatment, the nodule enlarged to $16 \mathrm{~mm}$ (Fig. 2). Because no other metastasis was found by systemic examination, it was diagnosed as a solitary metastasis, and we decided to remove it surgically. After sufficient informed consent concerning the surgery was obtained, thoracoscopic partial resection of the left lung was performed using the lipiodol-marking method. 
The diagnosis of pulmonary metastasis from BSCC of the esophagus was made after pathological observations (Fig. 3A and 3B). No adjuvant chemotherapy was administered because she was elderly patient. At present, one year after surgery, the patient is doing well without any recurrence.

\section{Discussion}

BSCC of the esophagus is a relatively rare variant. The incidences of BSCC differ according to reports, ranging from $0.77 \%$ to $5 \%$ in Asian groups ${ }^{1-6)}$ and from $6.9 \%$ to $11.3 \%$ in Western groups. ${ }^{7,8)}$ In 332 esophageal cancer cases who underwent surgical resection at our department between 1987 and 2010 nine cases (2.7\%) were diagnosed as BSCC. The incidence of BSCC in our hospital is similar to that in previous Asian reports. Wain, et al. initially reported the histological features of BSCC as small crowded cells with hyperchromatic nuclei, scant cytoplasm, small cystic spaces, and foci of tumor necrosis. ${ }^{9)}$

BSCC of the esophagus was previously regarded as a cancer with a very poor prognosis compared with squamous cell carcinoma (SCC) because it was characterized by high proliferative activity and high incidence of distant metastases. However, recent reports have suggested that the differences in prognosis between BSCC and conventional SCC remain controversial. Imamhasan, et al. have reported that patients with BSCC demonstrated significantly worse survival rates compared with patients with well-differentiated SCC in a stage-matched casecontrol study with 22 BSCC patients. ${ }^{1)}$ Chen, et al. also reported that the median survival time for patients with BSCC was significantly lower than that for patients with well-differentiated SCC; however, there were no significant differences between patients with BSCC and those with moderately or poorly differentiated SCC in a study of 26 BSCC patients. ${ }^{2)}$ Sarbia, et al. previously reported that the prognosis of patients with BSCC does not differ from that of patients with typical SCC in an evaluation of 17 BSCC patients. ${ }^{8)}$

Standard treatment strategy for BSCC has not yet been established because of the limited number of cases. At present, treatment for conventional SCC is usually used for the treatment of BSCC. Combined chemotherapy using cisplatin and 5-fluorouracil (5-FU) has been well established for conventional SCC, ${ }^{10)}$ whereas the efficacy of this regimen for BSCC has not yet been evaluated. Shibata, et al. reported a case of recurrent BSCC that showed good response to this regimen. ${ }^{11)}$ In contrast, Takemura, et al. analyzed the activity of the 5-FU-related enzymes in esophageal cancer tissue and they found that thymidylate synthase (TS) activity in BSCC was significantly higher than that in SCC. ${ }^{12)}$ The findings of the study by Takemura, et al. suggest that BSCC is more resistant to 5-FU treatment than SCC. TS-1 is an oral fluoropyrimidine formulation that combines tegafur, 5-chloro-2,4-dihydroxypyridine, and potassium oxonate. ${ }^{13)}$ Tegafur is a pro-drug that is metabolized to 5-FU in blood, and it is designed to enhance antitumor activity and reduce gastrointestinal toxicity compared with 5-FU. In our case, treatment with TS-1 was not effective for pulmonary metastasis. There is a possibility that TS activity affected the effects of TS- 1 treatment.

Through literature and database research, we found that no surgical case for pulmonary metastasis of BSCC has been reported until very recently. In 2012, three independent cases have been reported from Japan. ${ }^{12,14,15)}$ All three cases were solitary metastases, and all patients have survived without postoperative recurrence. Some investigators have reported the benefit of surgical resection for pulmonary metastasis of SCC in the esophagus. ${ }^{16-18)}$ Ichikawa, et al. retrospectively analyzed 23 resected cases of metachronous pulmonary metastasis from esophageal SCC and reported that operative intervention is acceptable, except in cases with antecedent extrapulmonary metastasis because of its unfavorable prognosis. In our case, we decided to perform surgical resection because it was a solitary metastasis and was refractory to chemotherapy. Although the first site of recurrence was the mediastinal lymph node, we thought the mediastinal lymph node disease control was effective because we obtained a twoyear disease-free interval after irradiation. Because the primary esophageal cancer was in an advanced stage, a close follow-up observation should be conducted.

In conclusion, we managed a case of a resected solitary pulmonary metastasis of BSCC of the esophagus. At present, there is no established treatment strategy for pulmonary metastases from BSCC of the esophagus because of the limited number of cases. Additional cases are needed to determine the treatment method.

\section{Disclosure Statement}

Takayuki Kosaka and other co-authors have no conflict of interest. 


\section{References}

1) Imamhasan $A$, Mitomi H, Saito T, et al. Immunohistochemical and oncogenetic analyses of the esophageal basaloid squamous cell carcinoma in comparison with conventional squamous cell carcinomas. Hum Pathol 2012; 43: 2012-23.

2) Chen SB, Weng HR, Wang G, et al. Basaloid squamous cell carcinoma of the esophagus. J Cancer Res Clin Oncol 2012; 138: 1165-71.

3) Kobayashi Y, Nakanishi Y, Taniguchi H, et al. Histological diversity in basaloid squamous cell carcinoma of the esophagus. Dis Esophagus 2009; 22: 231-8.

4) Li TJ, Zhang YX, Wen J, et al. Basaloid squamous cell carcinoma of the esophagus with or without adenoid cystic features. Arch Pathol Lab Med 2004; 128 : 1124-30.

5) Ohashi K, Horiguchi S, Moriyama S, et al. Superficial basaloid squamous carcinoma of the esophagus. A clinicopathological and immunohistochemical study of 12 cases. Pathol Res Pract 2003; 199: 713-21.

6) Cho KJ, Jang JJ, Lee SS, et al. Basaloid squamous carcinoma of the oesophagus: a distinct neoplasm with multipotential differentiation. Histopathology 2000; 36: 331-40.

7) Bellizzi AM, Woodford RL, Moskaluk CA, et al. Basaloid squamous cell carcinoma of the esophagus: assessment for high-risk human papillomavirus and related molecular markers. Am J Surg Pathol 2009; 33: 1608-14.

8) Sarbia M, Verreet P, Bittinger F, et al. Basaloid squamous cell carcinoma of the esophagus: diagnosis and prognosis. Cancer 1997; 79: 1871-8.

9) Wain SL, Kier R, Vollmer RT, et al. Basaloid-squamous carcinoma of the tongue, hypopharynx, and larynx: report of 10 cases. Hum Pathol 1986; 17: 1158-66.

10) Hayashi K, Ando N, Watanabe H, et al. Phase II evaluation of protracted infusion of cisplatin and 5-fluorouracil in advanced squamous cell carcinoma of the esophagus: a Japan Esophageal Oncology Group (JEOG) Trial (JCOG9407). Jpn J Clin Oncol 2001; 31: 419-23.

11) Shibata $Y$, Baba E, Ariyama H, et al. Metastatic basaloid-squamous cell carcinoma of the esophagus treated by 5 -fluorouracil and cisplatin. World J Gastroenterol 2007; 13: 3634-7.

12) Takemura M, Morimura K, Yoshida K, et al. [Four resected cases with basaloid carcinoma of esophaguscomparison of 5-FU-related enzymes (thymidylate synthase (TS), dihydropyrimidine dehydrogenase (DPD), orotate phosphoribosyl transferase (OPRT)) between basaloid carcinoma and squamous cell carcinoma]. Gan To Kagaku Ryoho 2010; 37: 2143-6 (in Japanese).

13) Okamoto I, Fukuoka M. S-1: a new oral fluoropyrimidine in the treatment of patients with advanced non-small-cell lung cancer. Clin Lung Cancer 2009; 10: 290-4.

14) Shibasaki H, Yoshino A, Okada N, et al. A case of basaloid carcinoma of the esophagus with a solitary lung metastasis for which thoracoscopic partial lung resection was performed. Gen Thorac Cardiovasc Surg 2012; 60: 673-9.

15) Yoshino A, Shibasaki H, Okada N, et al. [Lung metastasis of basaloid-squamous cell carcinoma of the esophagus; report of a case]. Kyobu Geka 2012; 65: 335-7 (in Japanese).

16) Ichikawa $H$, Kosugi $S$, Nakagawa $S$, et al. Operative treatment for metachronous pulmonary metastasis from esophageal carcinoma. Surgery 2011; 149: 164-70.

17) Shiono S, Kawamura M, Sato T, et al. Disease-free interval length correlates to prognosis of patients who underwent metastasectomy for esophageal lung metastases. J Thorac Oncol 2008; 3: 1046-9.

18) Chen F, Sato K, Sakai H, et al. Pulmonary resection for metastasis from esophageal carcinoma. Interact Cardiovasc Thorac Surg 2008; 7: 809-12. 